\title{
Combined inhibition of DNA methylation and histone acetylation enhances gene re-expression and drug sensitivity in vivo
}

\author{
N Steele', P Finn', R Brown' and JA Plumb*,1 \\ 'Centre for Oncology and Applied Pharmacology, University of Glasgow, CRUK Beatson Laboratories, Garscube Estate, Glasgow G6I IBD, UK; \\ ${ }^{2}$ TopoTarget Prolifix Ltd, Abingdon, Oxford OXI 4 4RY, UK
}

Histone deacetylation and DNA methylation have a central role in the control of gene expression in tumours, including transcriptional repression of tumour suppressor genes and genes involved in sensitivity to chemotherapy. Treatment of cisplatinresistant cell lines with an inhibitor of DNA methyltransferases, 2-deoxy-5'azacytidine (decitabine), results in partial reversal of DNA methylation, re-expression of epigenetically silenced genes including $\mathrm{hMLHI}$ and sensitisation to cisplatin both in vitro and in vivo. We have investigated whether the combination of decitabine and a clinically relevant inhibitor of histone deacetylase activity (belinostat, PXDIOI) can further increase the re-expression of genes epigenetically silenced by DNA methylation and enhance chemosensitisation in vivo at well-tolerated doses. The cisplatin-resistant human ovarian cell line A2780/cp70 has the hMLHI gene methylated and is resistant to cisplatin both in vitro and when grown as a xenograft in mice. Treatment of A2780/cp70 with decitabine and belinostat results in a marked increase in expression of epigenetically silenced MLHI and MAGE-AI both in vitro and in vivo when compared with decitabine alone. The combination greatly enhanced the effects of decitabine alone on the cisplatin sensitivity of xenografts. As the dose of decitabine that can be given to patients and hence the maximum pharmacodynamic effect as a demethylating agent is limited by toxicity and eventual re-methylation of genes, we suggest that the combination of decitabine and belinostat could have a role in the efficacy of chemotherapy in tumours that have acquired drug resistance due to DNA methylation and gene silencing.

British Journal of Cancer (2009) 1 00,758-763. doi:I0.1038/sj.bjc.6604932 www.bjcancer.com

(c) 2009 Cancer Research UK

Keywords: DNA methylation; MLHI; drug sensitivity; decitabine; histone deacetylase inhibitor

Epigenetic inactivation of genes crucial for control of normal cell growth is a hallmark of cancer cells (Hanahan and Weinberg, 2000). These epigenetic mechanisms include crosstalk between DNA methylation, histone modification and other components of chromatin higher order structure leading to regulation of gene transcription. The transfer of a methyl group to the carbon 5 position of cytosines, almost always in the context of $\mathrm{CpG}$ dinucleotides, is the only known epigenetic modification of DNA itself in mammalian cells. Many tumours show increased methylation of $\mathrm{CpG}$ islands, $\mathrm{CpG}$ rich regions of DNA usually although not exclusively associated with gene promoters, which is associated with epigenetic silencing (Brown and Strathdee, 2002). $\mathrm{CpG}$ islands aberrantly methylated in tumours are associated with silencing of genes involved in control of the cell cycle, apoptosis and drug sensitivity as well as tumour suppressor genes (Brown and Plumb, 2004).

Epigenetic silencing occurring during tumour development has the potential to affect the chemo-sensitivity of tumour cells and how tumours will respond to chemotherapy (Teodoridis et al, 2005). One example of this is epigenetic inactivation of the CpG island at the DNA repair gene, MGMT and response of glioma to monofunctional alkylating agents such as temozolomide (Hegi et al, 2005). DNA methylation and epigenetic silencing of tumour

*Correspondence: Dr JA Plumb; E-mail: Jane.Plumb@beatson.gla.ac.uk Received 7 October 2008; revised 12 January 2009; accepted I5 January 2009 cells can also be selected for during chemotherapy and may be an important driving force behind acquired drug resistance (Glasspool et al, 2006). The MLH1 protein, part of the human DNA mismatch repair system, has been shown to be important in determining sensitivity to a number of important chemotherapeutic agents including alkylating agents and cisplatin (Fink et al, 1998; Brown, 1999). Loss of mismatch repair due to methylation of the $h M L H 1$ gene promoter results in resistance to cisplatin in cell lines in vitro and in human tumour xenografts in vivo (Plumb et al, 2000). Methylation of the $h M L H 1$ gene promoter is observed in many tumour types (Strathdee et al, 1999; Gifford et al, 2004) and loss of MLH1 expression is associated with clinical drug resistance in ovarian cancer (Samimi et al, 2000).

There is considerable interest in the potential to use epigenetic therapies in combination with existing chemotherapeutic agents, both for improving initial tumour response and for overcoming acquired drug resistance. We have previously shown that treatment of ovarian and colon cell lines with the DNA hypomethylating agent 2-deoxy-5'azacytidine (decitabine, DAC) results in partial reversal of DNA methylation, re-expression of methylated loci such as $h M L H 1$ and sensitisation to cisplatin and carboplatin both in vitro and in vivo (Plumb et al, 2000). In studies of human tumour xenografts in mice we were able to demonstrate that decitabine treatment reduced the level of methylation of the hMLH1 gene promoter and that this was associated with reexpression of MLH1 in a small proportion of the tumour cells at doses that clearly conferred increased sensitisation and were well 
tolerated. In a phase 1 clinical trial of decitabine in combination with carboplatin in advanced solid tumours, a reduction in methylcytosine content of PBMCs was observed that was comparable to that observed in mice where chemo-sensitisation of xenografts occurred (Appleton et al, 2007). However, limited demethylation of tumour was observed. The dose limiting toxicity of decitabine was identified as myelosuppression and this toxicity plus the limited demethylation in tumours and eventual remethylation of genes may limit the clinical use of decitabine when used alone in solid tumours.

Baylin and co-workers have shown that the combination of the histone deacetylase (HDAC) inhibitor trichostatin A with decitabine is more effective in reactivating transcription of epigenetically silenced genes such as $h M L H 1$ in tumour cell lines than either drug alone (Cameron et al, 1999). The combination of a demethylating agent and an HDAC inhibitor has now been examined in clinical trials of haematological malignancies. However, in solid tumours it may be that epigenetic therapies may be more effective when used in combination with cytotoxic agents. We have therefore investigated whether it is possible to use a low, non-toxic dose of decitabine in combination with an inhibitor of histone deacetylase activity, belinostat (PXD101), to enhance the level of re-expression of epigenetically silenced genes in drug-resistant A2780/cp70 both in vitro and in vivo and whether this combination enhances chemo-sensitisation of xenografts.

\section{MATERIALS AND METHODS}

\section{Cell lines}

Cell line A2780/cp70 is an in vitro derived cisplatin-resistant variant of the ovarian cancer cell line A2780 originally obtained from Dr RF Ozols (Fox Chase Cancer Centre, Philadelphia, PA, USA). Cells were grown in RPMI-1640 supplemented with glutamine ( $2 \mathrm{~mm})$ and FCS (10\%). A2780/cp70 is mismatch repair deficient and does not express MLH1 due to hypermethylation of the $h M L H 1$ gene promoter (Strathdee et al, 1999) as well as having a number of other loci hypermethylated (Leu et al, 2003).

\section{Protein expression}

For western blotting cells were grown in $25 \mathrm{~cm}^{2}$ flasks and exposed to drugs as specified. Cells were harvested with trypsin/EDTA and washed two times with ice cold PBS. They were resuspended in $200 \mu \mathrm{l}$ of lysis buffer ( $50 \mathrm{~mm}$ Hepes pH 7.0, $250 \mathrm{~mm} \mathrm{NaCl}, 0.5 \%$ NP40) supplemented with protease inhibitors (Complete from Roche Diagnostics Ltd, Lewes, UK) and incubated on ice for $20 \mathrm{~min}$. Samples were centrifuged at $12000 \mathrm{~g}$ for $5 \mathrm{~min}$ at $4{ }^{\circ} \mathrm{C}$ to remove debris. Supernatant proteins $(20 \mu \mathrm{g})$ were separated by the NuPage electrophoresis system (Invitrogen, Paisley, UK) on 4$12 \%$ Bis-Tris gels with 4-morpholinepropanesulfonic acid SDS running buffer. The 'Novex Xcell II' blotting apparatus (Invitrogen) was used to transfer proteins onto Immobilon polyvinylidene difluoride membrane (Millipore, Watford, UK). The membrane was blocked for $1 \mathrm{~h}$ in Tris-buffered saline containing $0.02 \%$ Tween 20 and $5 \%$ powdered milk and then incubated overnight at $4{ }^{\circ} \mathrm{C}$ with the primary antibody (MLH1 from Pharmingen, BD UK Ltd, Oxford, UK, MAGE-A1 from Neomarkers, Lab Vision Ltd, Cambridge, UK). The membrane was then washed and incubated for $1 \mathrm{~h}$ at room temperature with the secondary antibody (sheep anti-mouse HRP, Amersham, GE Healthcare, Amersham, UK). After washing, the membrane protein bands were visualised by enhanced chemiluminescence (ECL, Amersham). Band intensity was quantified by densitometry (GS-800, Bio-Rad, Hemel Hempstead, UK).

For immunohistochemistry, tumours were fixed in neutralbuffered formalin and processed as previously described (Plumb et al, 2000).

\section{Human tumour xenografts}

Animal studies were carried out under an appropriate United Kingdom Home Office Project Licence and all work conformed to the UKCCR Guidelines for the welfare of animals in experimental neoplasia. Monolayer cultures were harvested with trypsin/EDTA and resuspended in PBS. About $10^{7}$ cells were injected subcutaneously into the right flank of athymic nude mice (CD1 $\mathrm{nu} / \mathrm{nu}$ mice from Charles River, Margate, UK). After 7-10 days when the mean tumour diameter was at $\geqslant 0.5 \mathrm{~cm}$, animals were randomised in groups of six for experiments. Standard sterile clinical formulations of cisplatin (Western Infirmary Pharmacy, Glasgow) decitabine (Supergen, Dublin, California, CA, UK) and belinostat (TopoTarget, Abingdon, UK) were used. Where specified, mice were pretreated with decitabine 6 days before cisplatin $\left(6 \mathrm{mg} \mathrm{kg}^{-1}\right.$ intraperitoneally), when tumours were just visible. Decitabine $\left(5 \mathrm{mg} \mathrm{kg}^{-1}\right)$ was administered intraperitoneally at 10:00, 13:00 and $16: 00$ hours (total dose $15 \mathrm{mg} \mathrm{kg}^{-1}$ per mouse). Belinostat $\left(40 \mathrm{mg} \mathrm{kg}^{-1}\right)$ was administered intraperitoneally 3 days before cisplatin where specified. Mice were weighed daily and tumour volumes were estimated by caliper measurements assuming spherical geometry (volume $\left.=d^{3} \times \pi / 6\right)$.

\section{Pyrosequencing}

The methylation status of specific cytosine residues in the MAGEA1 gene promoter was determined following bisulphite modification of DNA extracted from tumours. Tumours were removed from mice and snap frozen in liquid nitrogen. Frozen tumours were powdered in a 'Mikro-Dismembrator' homogeniser and DNA extracted with a BACC2 Nucleon extraction kit (Nucleon). Bisulphite modification was carried out with the CpGenome DNA modification kit (Chemicon International, Millipore, Watford, UK) according to the manufacturer's instructions. The modified DNA was amplified by PCR with primers chosen to bracket the CpG island of the MAGE-A1 gene promoter (forward PCR: $5^{\prime}$-TTTTTATTTTTATTTAGGTAGGAT- $3^{\prime}$ and reverse PCR: Biotin$5^{\prime}$-TCTAAAAACAACCCAAACTAAAAC- $\left.3^{\prime}\right)$. The PCR was carried out in $50 \mu \mathrm{l}$ volumes containing $2 \mathrm{U}$ Faststart Taq polymerase, $10 \times$ Faststart Buffer (Roche), $10 \mathrm{~mm}$ dNTPs (Applied Biosystems, Warrington, UK), $3.5 \mathrm{~mm} \mathrm{MgCl}_{2}$ (Roche), oligonucleotides (Biomers, www.biomers.net) at $1 \mu \mathrm{M}$ and $2 \mu \mathrm{l}$ of modified DNA template. A 40- $\mu$ l PCR product was used for pyrosequencing according to the manufacturer's instruction. Sixteen picomoles of the sequencing primers ( $5^{\prime}$-TGTTGTTAGTTTTGGTTTAT- $\left.3^{\prime}\right)$ were applied to detect the presence or absence of methylation.

\section{RESULTS}

\section{Re-expression of MLH1 and MAGE-A1 in vitro by decitabine and belinostat}

Treatment of MLH1 negative A2780/cp70 cells on days 1 and 2 with decitabine results in a dose-dependent re-expression of MLH1 as measured by western blot 3, 6 and 9 days after the start of treatment (Figure 1A). Belinostat treatment alone had no detectable effect on MLH1 levels. Treatment with decitabine on day 1 and with both decitabine and belinostat on day 2 results in a marked increase in MLH1 expression compared to treatment with decitabine alone on days 1 and 2. Re-expression of MLH1 was transient following treatment with decitabine at $0.1 \mu \mathrm{m}$ (Figure 1B) but was more sustained at $0.2 \mu \mathrm{M}$ (Figure 1C). Addition of belinostat increased the level of expression of MLH1 but did not alter the time course of re-expression or re-silencing. Decitabine treatment also induced re-expression of MAGE-A1 and again the expression was enhanced by the addition of belinostat. Re-expression of MAGE-A1 was transient at both concentrations 

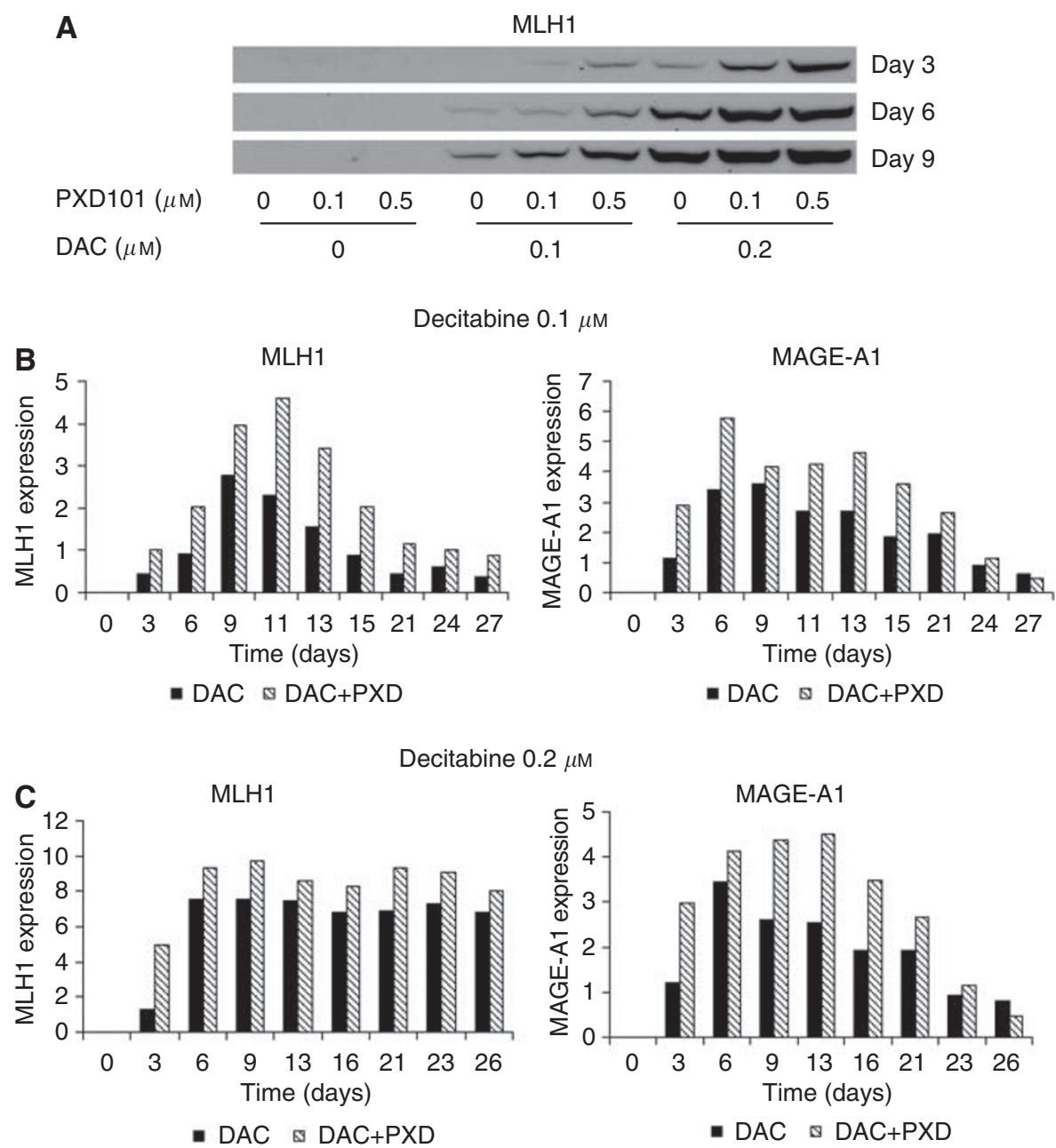

Figure I (A) MLHI expression determined by western blotting of A2780/cp70 cell lysates prepared at various times after treatment of cells with DAC either alone or in combination with belinostat (PXDIOI). Cells were treated for $48 \mathrm{~h}$ (days I and 2) with decitabine with belinostat added for the second $24 \mathrm{~h}$ (day 2). MLHI and MAGE-Ia expression determined by western blotting and quantified by densitometry at various times after treatment of A2780/cp70 cells with either decitabine (B) $0.1 \mu \mathrm{M},(\mathbf{C}) 0.2 \mu \mathrm{M}$; filled bars) or decitabine and belinostat (0.I $\mu \mathrm{M}$; hatched bars). Results shown are representative of one of three experiments.

of decitabine and belinostat and did not alter the time course of MAGE-A1 re-expression or re-silencing (Figure 1B and C).

\section{Re-expression of MLH1 and MAGE-A1 in vivo by decitabine and belinostat}

Treatment of mice with decitabine induces re-expression of MLH1 in A2780/cp70 xenografts and expression is maximal by about day 9 (Figure 2A and B). A similar time course is observed for MAGEA1 expression (Figure 2C). Belinostat alone has no detectable effect on MLH1 and MAGE-A1 expression. The combination of decitabine and belinostat produces a marked increase in the level of re-expression of both MLH1 and MAGE-A1 to a greater extent than that achieved with decitabine alone (Figure 2). Gene reexpression is detectable by immunocytochemistry in only about $10 \%$ of cells and these cells appear in clusters following decitabine and belinostat treatment (Figure 2A, day 12).

\section{Methylation of MAGE-A1}

Cytosine methylation was examined at $3 \mathrm{CpG}$ sites within the MAGEA1 gene promoter. At each site the level of methylation was reduced by decitabine treatment but there was no further reduction following treatment with decitabine and belinostat in combination (Figure 3). Although only between 6 and 20\% demethylation is observed at these sites it should be noted that this will be an average throughout the cell population and only those cells, which are proliferating will incorporate decitabine and become demethylated. Global 5-methyl-2'-deoxycytidine levels in DNA from the tumours taken on day 6 was measured by HPLC (Appleton et al, 2007). Decitabine treatment reduced cytosine methylation (5-methyl-2' -deoxycytidine as a percentage of total $2^{\prime}$-deoxycytidine) from $3.43 \pm 0.16$ in the control tumours to $2.78 \pm 0.05(n=3$, $P<0.01)$ and this was not significantly different from the levels observed in the tumours taken from mice treated with decitabine in combination with belinostat $(2.92 \pm 0.12, n=3)$ Belinostat treatment had no effect on 5-methyl-2'-deoxycytidine levels $(3.70 \pm 0.28, n=3)$.

\section{Effects of decitabine and belinostat pre-treatment on drug sensitivity}

A2780/cp70 is resistant to the maximum-tolerated dose of cisplatin in vivo. Treatment with decitabine or belinostat either alone or in combination has no effect on tumour growth and belinostat did not sensitise tumours to cisplatin. Pre-treatment of the mice with decitabine 6 days before treatment with cisplatin results in a significant growth delay and this effect is enhanced by the 
A
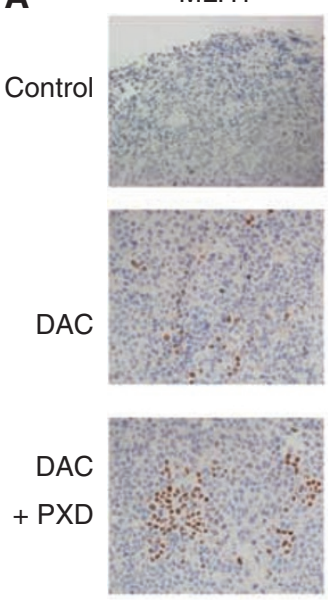

MAGE-A1
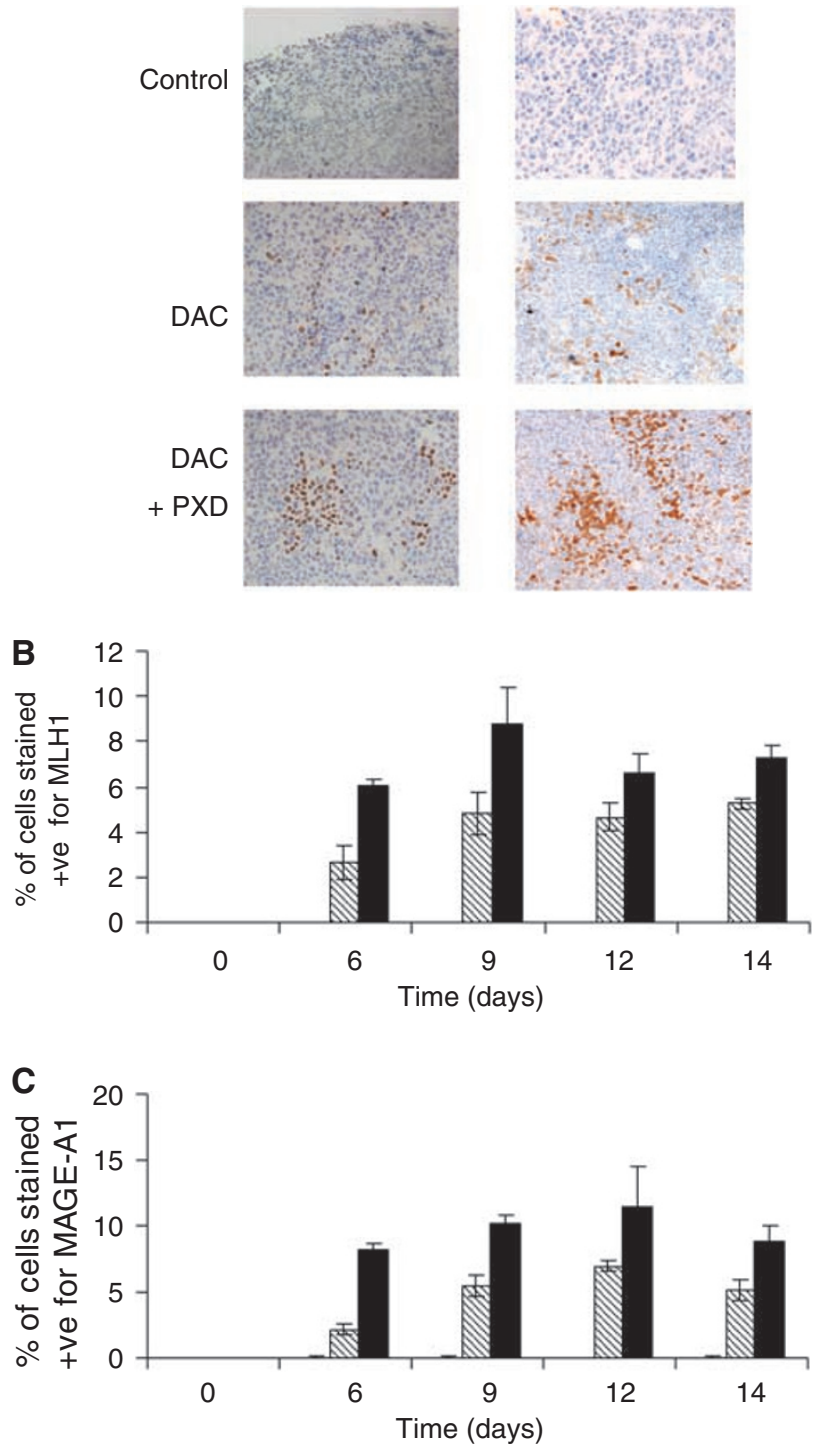

Figure 2 (A) MLHI and MAGE-IA expression detected by immunohistochemistry in A2780/cp70 xenografts 12 days after treatment with decitabine $\left(5 \mathrm{mg} \mathrm{kg}^{-1} \times 3\right.$ on day 0$)$ alone or followed by belinostat (40 $\mathrm{mg} \mathrm{kg}^{-1}$ on day 3). (B) MLHI and MAGE- la expression at various times after treatment with belinostat (open bars), decitabine (hatched bars) or decitabine and belinostat (filled bars). (C) Expression is quantified as the percentage of cells that stain positive $(+v e)$.

combination of decitabine and belinostat (Figure 4A). The treatments were well tolerated by the mice and there was no significant effect on body weight (Figure 4B).

\section{DISCUSSION}

We have shown clearly that the combination of low doses of decitabine and belinostat results in re-expression of epigenetically silenced genes and that when used in vivo in mice the combination can sensitise drug-resistant tumours to cisplatin more effectively than either drug alone.

We have already established that decitabine can be used to sensitise drug-resistant tumours to a number of clinically relevant cytotoxic drugs including cisplatin, carboplatin, epirubicin and temozolomide (Plumb et al, 2000). The inclusion of pharmacodynamic measurements in a phase 1 trial of decitabine and
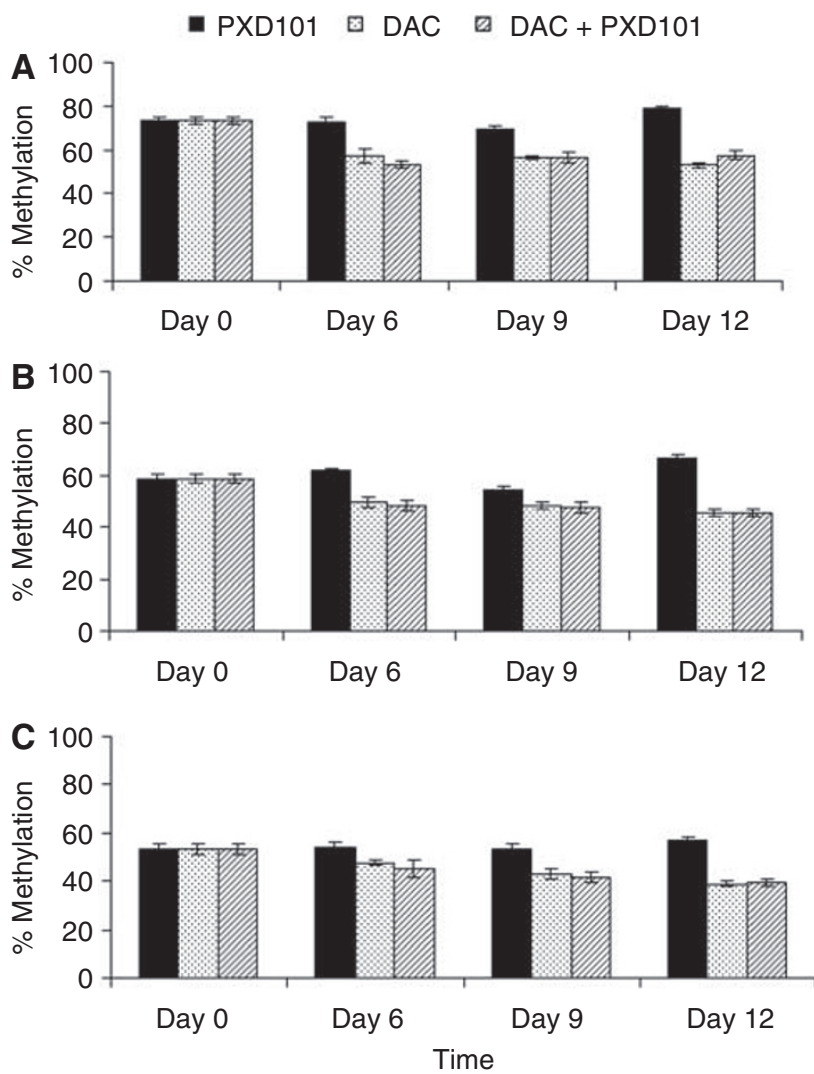

Figure 3 Pyrosequencing analysis of methylation of three $\mathrm{CpG}$ sites within a CPG island in the MAGE-I a gene promoter in A2780/cp70 xenografts. Mice were treated with decitabine alone on day 0 $\left(5 \mathrm{mg} \mathrm{kg}^{-1} \times 3\right.$; stippled bars), belinostat alone on day $3\left(40 \mathrm{mg} \mathrm{kg}^{-1}\right.$; filled bars) or with the combination of decitabine on day 0 and belinostat on day 3 (hatched bars). Tumours were removed and cytosine methylation measured on day 0 (untreated) and on days 6, 9 and 12. Cytosine methylation at three $\mathrm{CPG}$ sites within the MAGE-AI promoter (CpG 17 (A), CpG 18 (B) and CpG $19(\mathbf{C})$ ) was determined.

carboplatin has enabled us to show that decitabine can induce in patients the levels of demethylation in surrogate PBMCs seen in our mouse studies at doses that cause chemo-sensitisation (Appleton et al, 2007). However, it is also clear from the phase 1 trial that demethylation in PBMCs by decitabine is limited by the myelosuppressive activity of the drug. The level of demethylation observed in tumours was limited and heterogeneous between patients. In order to potentially increase the reversal of epigenetic silencing by decitabine, we have examined the addition of an HDAC inhibitor on re-expression of epigenetically silenced genes and chemo-sensitisation. Belinostat alone has no effect on MLH1 expression and this is consistent with the observation that histone deacetylase inhibitors cannot induce the expression of genes silenced due to promoter methylation (Suzuki et al, 2002). From the results with the cell line it is clear that belinostat can enhance the effects of decitabine on gene re-expression (Figure 1). Although belinostat increases the level of re-expression of both MLH1 and MAGE-A1 it does not appear to alter the kinetics of re-expression. This is consistent with the observation that the HDAC inhibitor 4-phenylbutyric acid does not inhibit re-silencing of p16 after decitabine treatment (Egger et al, 2007). Re-expression of MAGE$\mathrm{A} 1$ is transient. It can be detected by 3 days after treatment but is lost after about 26 days. Re-expression of MLH1 is also transient at lower concentrations of decitabine $(0.1 \mu \mathrm{M})$, but is more sustained at the higher concentration $(0.2 \mu \mathrm{M})$ such that it remains detectable after 26 days. This may reflect the long half-life of the protein or 

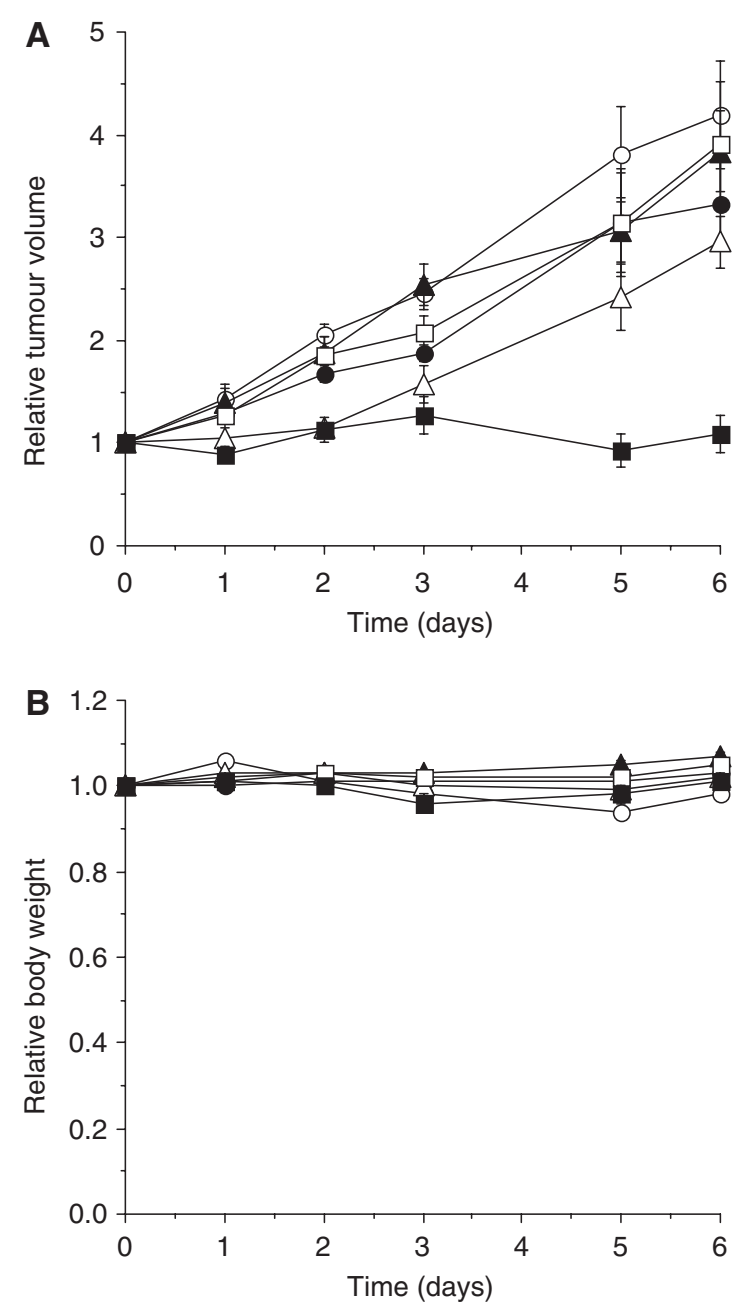

Figure 4 The effect of decitabine and belinostat pre-treatment on the drug sensitivity of $A 2780 / \mathrm{cp} 70$ xenografts. Mice were treated with decitabine $\left(5 \mathrm{mg} \mathrm{kg}^{-1} \times 3\right) 6$ days before cisplatin treatment (day -6$)$ and with belinostat $\left(40 \mathrm{mg} \mathrm{kg}^{-1}\right) 3$ days before treatment with cisplatin $($ day -3$)$. Cisplatin $\left(6 \mathrm{mg} \mathrm{kg}^{-}\right)$was administered on day 0 . The groups are control ( ; untreated); cisplatin alone $\left(0 ; 6 \mathrm{mg} \mathrm{kg}^{-1}\right.$ on day 0$)$; decitabine alone $\left(\boldsymbol{\Lambda} ; 5 \mathrm{mg} \mathrm{kg}^{-1} \times 3\right.$ on day -6$)$; decitabine and cisplatin ( $\triangle$; decitabine on day -6 and cisplatin on day 0$)$; belinostat alone $\left(\square ; 40 \mathrm{mg} \mathrm{kg}^{-1}\right.$ on day $-3)$; decitabine and belinostat followed by cisplatin ( $\mathbf{\square}$; decitabine on day -6 , belinostat on day -3 and cisplatin on day 0 ). Results for $(\mathbf{A})$ tumour volumes and $(\mathbf{B})$ body weights are the means \pm s.e.m. of six mice.

may be due to a slower rate of gene re-methylation at the higher dose of decitabine.

To study the combination in human tumour xenografts we used the same schedule for decitabine that was shown to sensitise the tumours to cisplatin (Plumb et al, 2000) and attempted to improve on this response. Initial studies investigated the effects on gene reexpression and we have shown that a single dose of belinostat administered 3 days after decitabine treatment results in an increase in expression of both MLH1 and MAGE-A1 to a level greater that is seen with decitabine alone (Figure 2). MLH1 and MAGE-A1 gene reexpression is detected in about $6 \%$ of cells following treatment with decitabine and increases to about $10-12 \%$ when mice are treated with the combination of decitabine and belinostat. The apparent clustering of cells that re-express MLH1 and MAGE-A1 in the xenografts could represent areas of active proliferation within the tumours, which would be consistent with decitabine being incorporated into DNA during S-phase only and cell proliferation being required for demethylation. For the MAGE-A1 gene promoter decitabine treatment results in a decrease in the methylation of all three CpG sites studied (Figure 3). However, there is no further reduction in methylation following addition of belinostat to decitabine, which suggests that the enhanced gene expression observed with the combination is not due to direct effects on gene methylation. A study of the combination of decitabine and trichostatin A on MLH1 expression also concluded that the effect of the HDAC inhibitor was not due to a further reduction in DNA methylation (Cameron et al, 1999). It is possible that the HDAC inhibitor allows increased access of the transcription factors to the demethylated gene as a result of increased levels of histone acetylation and the resulting chromatin remodelling (Egger et al, 2007).

Re-expression of MLH1 is clearly apparent by 6 days after treatment and is maximal by about 9 days. As A2780/cp70 is a rapidly growing tumour, we treated with the cytotoxic drug as early as possible after decitabine treatment (day 6). This is the schedule used previously for decitabine alone (Plumb et al, 2000). A2780/cp70 xenografts are resistant to cisplatin. However, treatment with decitabine sensitises the tumours to cisplatin and the growth delay is further enhanced by the addition of belinostat (Figure 4). These results give clear support to the proposal to use decitabine in combination with a histone deacetylase inhibitor to enhance the chemo-sensitisation observed with decitabine alone.

Neither decitabine, belinostat nor the combination had any effect on tumour growth. This is not surprising as we have not attempted to use these drugs in the optimal schedule for antitumour activity. We have already shown that A2780/cp70 is sensitive to belinostat when mice are treated daily for 7 days (Plumb et al, 2003). As the aim was to combine the epigenetic therapies with a cytotoxic drug we have intentionally used low, non-toxic doses of the agents. Although decitabine treatment results in a reduced MAGE-A1 methylation in PBMCs the gene is not re-expressed and this may be due to a lack of the necessary transcriptional activators (Karpf et al, 2004). Few studies have examined the effects of demethylating agents on normal cells; however, there is some evidence that fewer genes become demethylated than in tumour cells (Liang et al, 2002). This suggests that epigenetic therapies will not necessarily be associated with genome-wide effects in normal tissues. Furthermore, the combination of a low dose of decitabine and the HDAC inhibitor phenylbutyrate has been shown to inhibit carcinogen-induced lung tumours in mice (Belinsky et al, 2004). This raises the possibility that in addition to sensitising drug-resistant tumours to chemotherapy the epigenetic therapy might also protect the normal tissues from some of the damage caused by the cytotoxic agent.

As the dose of decitabine that can be given to patients and hence the maximum pharmacodynamic effect as a demethylating agent is limited by toxicity and eventual re-methylation of genes, we suggest that the combination of decitabine and belinostat could have a role in increasing the efficacy of chemotherapy in tumours that have acquired drug resistance due to DNA methylation and gene silencing.

\section{REFERENCES}

Appleton K, Mackay HJ, Judson I, Plumb JA, McCormick C, Strathdee G, Lee C, Barrett R (2007) Phase I and pharmacodynamic trial of the DNA

methyltransferase inhibitor decitabine and carboplatin in solid tumours. J Clin Oncol 25: 4603-4609 
Belinsky SA, Klinge DM, Stidley CA, Issa JP, Herman JG, March $\mathrm{TH}$, Baylin SB (2004) Inhibition of DNA methylation and histone deacetylation prevents murine lung cancer. Cancer Res 63: $7089-7093$

Brown R (1999) Mismatch repair deficiency, apoptosis and drug resistance. In Apoptosis and Cancer Chemotherapy, Hickman JA, Dive C (eds), Vol. 5 , pp 69-86. Humana Press: Totowa, NJ

Brown R, Plumb JA (2004) Demethylation of DNA by decitabine in cancer chemotherapy. Expert Rev Anticancer Ther 4: 501-510

Brown R, Strathdee G (2002) Epigenomics and epigenetic therapy of cancer. Trends Mol Med 8(Suppl): S43-S48

Cameron EE, Bachman KE, Myohanen S, Herman JG, Baylin SB (1999) Synergy of demethylation and histone deacetylase inhibition in the re-expression of genes silenced in cancer. Nat Genet 21: $103-107$

Egger G, Aparicio AM, Escobar SG, Jones PA (2007) Inhibition of histone deacetylation does not block resilencing of p16 after 5-aza-2'-deoxycytidine treatment. Cancer Res 67: 346-353

Fink D, Aebi S, Howell SB (1998) Role of DNA mismatch repair in drug resistance. Clin Cancer Res 4: 1-6

Gifford G, Paul J, Kaye SB, Vasey PA, Brown R (2004) The acquisition of hMLH1 methylation in plasma DNA following chemotherapy predicts poor survival for ovarian cancer patients. Clin Cancer Res 10: $4420-4426$ Glasspool RM, Teodoridis JM, Brown R (2006) Epigenetics as a mechanism driving polygenic clinical drug resistance. Br J Cancer 94: 1087-1092

Hanahan D, Weinberg RA (2000) The hallmarks of cancer. Cell 100: 57-70 Hegi ME, Diserens AC, Gorlia T, Hamou MF, de Tribolet N, Weller M, Kros JM, Hainfellner JA, Mason W, Mariani L, Bromberg JE, Hau P, Mirimanoff RO, Cairncross JG, Janzer RC, Stupp R (2005) MGMT gene silencing and benefit from temozolomide in glioblastoma. $N$ Engl J Med 352: $997-1003$

Karpf AR, Lasek AW, Ririe TO, Hanks AN, Grossman D, Jones DA (2004) Limited gene activation in tumor and normal epithelial cells treated with the DNA methyltransferase inhibitor 5-aza-2'-deoxycytidine. Mol Pharmacol 65: 18-27

Leu YW, Rahmatpanah F, Shi H, Wei SH, Liu JC, Yan PS, Huang TH (2003) Double RNA interference of DNMT3b and DNMT1 enhances DNA demethylation and gene reactivation. Cancer Res 63: 6110-6115

Liang G, Gonzales FA, Jone PA, Orntoft TF, Thykjaer T (2002) Analysis of gene induction in human fibroblasts and bladder cancer cells exposed to the methylation inhibitor 5-aza-2'-deoxycytidine. Cancer Res 62: 961- 966

Plumb JA, Finn PW, Williams RJ, Bandarra MJ, Romero MR, Watkins CJ, LaThangue NB, Brown R (2003) Pharmacodynamic response and inhibition of growth of human tumour xenografts by the novel histone deacetylase inhibitor PXD101. Mol Cancer Ther 2: 721-728

Plumb JA, Strathdee G, Sludden J, Kaye SB, Brown R (2000) Reversal of drug resistance in human tumour xenografts by $2^{\prime}$-deoxy-5-azacytidineinduced demethylation of the hMLH1 gene promoter. Cancer Res 60: $6039-6044$

Samimi G, Fink D, Varki NM, Husain A, Hoskins WJ, Alberts DS, Howell SB (2000) Analysis of MLH1 and MSH2 expression in ovarian cancer before and after platinum drug-based chemotherapy. Clin Cancer Res 6: $1415-1421$

Strathdee G, MacKeen MJ, Illand M, Brown R (1999) A role for methylation of the $h M L H 1$ promoter in loss of hMLH1 expression and drug resistance in ovarian cancer. Oncogene 18: 2335-2341

Suzuki H, Gabrielson E, Chen W, Anbazhagan R, van Engeland M, Weijenberg MP, Herman JG, Baylin SB (2002) A genomic screen for genes upregulated by demethylation and histone deacetylase inhibition in human colorectal cancer. Nat Genet 31: $141-149$

Teodoridis JM, Hall J, Marsh S, Kannall HD, Smyth C, Curto J, Siddiqui N, Gabra H, McLeod HL, Strathdee G, Brown R (2005) CpG island methylation of DNA damage response genes in advanced ovarian cancer. Cancer Res 65: $8961-8967$ 